\title{
Aptitude physique versus adiposité : aspects physiopathologiques et impacts cardio-métaboliques chez le sujet adulte non diabétique
}

\author{
Fitness versus fatness: Pathophysiological aspects \\ and metabolic consequences in non-diabetic adults
}

\author{
N. Esser, N. Paquot, A. J. Scheen \\ Université de Liège, \\ Service de diabétologie, \\ nutrition et maladies métaboliques, \\ Département de médecine, \\ CHU Liège, Sart Tilman, \\ Liège, Belgique.
}

\begin{abstract}
Résumé
L'excès de masse grasse (adiposité), surtout abdominale, induit des effets cardiométaboliques néfastes, alors que l'exercice musculaire et une bonne aptitude physique exercent globalement une influence favorable. Les effets délétères d'un excès de masse grasse (fatness) pourraient donc être contrecarrés par la pratique régulière d'exercices aboutissant à une bonne forme physique (fitness). Cet article analyse d'abord les différents mécanismes physiopathologiques par lesquels l'exercice physique produit des effets bénéfiques chez la personne avec excès pondéral et fait le distinguo entre la pratique d'une activité physique (exercice musculaire) stricto sensu et aptitude physique (fitness). Ensuite, il décrit les études les plus importantes ayant analysé les relations entre le niveau d'aptitude physique et le degré d'adiposité chez le sujet adulte en surpoids ou obèse non diabétique et leurs influences respectives sur le risque de survenue de troubles métaboliques (syndrome métabolique) et sur la mortalité, en particulier cardiovasculaire.
\end{abstract}

Mots-clés : Adiposité - obésité - physiopathologie - syndrome métabolique - aptitude physique - exercice.

\begin{abstract}
Summary
Excessive fat depots, especially abdominal adiposity, exert deleterious cardiometabolic effects whereas physical exercise and good cardiorespiratory fitness globally exert a favourable influence. Therefore, the negative effects of excessive fat mass ("fatness") might be counterbalanced by the positive effects of regular physical activity leading to high "fitness". The present article first analyzes the various pathophysiological mechanisms explaining why muscular exercise exerts beneficial effects in overweight people and attempts to separate the effects of physical activity from those due to fitness. Finally, it describes most important studies focusing on the relationships between "fitness" and "fatness" in non-diabetic overweight or obese adults and their respective influences on metabolic disturbances (metabolic syndrome) and on death rate, especially cardiovascular mortality.
\end{abstract}

Key-words: Adiposity - obesity - fatness - metabolic syndrome - fitness - obesity - pathophysiology - exercise.

\section{André J. Scheen}

Département de médecine

CHU Sart Tilman (B35)

$B-4000$ Liège

Belgique

andre.scheen@chu.ulg.ac.be 


\section{Introduction}

Comme nous l'avons déjà discuté [1], environ $20 \%$ des sujets obèses et au moins $50 \%$ des sujets en surpoids ne présentent pas d'anomalies métaboliques significatives. Ces sujets ont un profil particulier avec, notamment, une moindre adiposité abdominale et une plus grande proportion de graisse souscutanée périphérique. II apparaît également que ces sujets sont plus actifs sur le plan physique [1]. Dès lors, on peut faire l'hypothèse qu'une meilleure aptitude physique (fitness) pourrait être capable de contrecarrer, au moins en partie, les conséquences cardio-métaboliques de l'excès de masse grasse (fatness) et d'atténuer, voire annuler, la surmortalité cardiovasculaire (CV) associée à l'obésité [2-4]. Force est de constater, cependant, que l'excès de poids est souvent associé avec une plus grande sédentarité et une moins bonne aptitude physique [5]. La pratique régulière d'une activité physique devrait donc faire partie de toute stratégie de prise en charge préventive ou thérapeutique dans une optique d'amélioration globale de la santé [6], en particulier chez des personnes à risque, mais également chez les personnes obèses encore dépourvues de risque cardiométabolique [7].

Le but du présent travail est d'analyser les répercussions cardio-métaboliques respectives de l'obésité et de l'aptitude physique et, notamment, d'étudier si une bonne condition physique cardiorespiratoire, par la pratique régulière d'exercices musculaires, permet de limiter l'impact délétère, métabolique et cardiovasculaire, de l'excès de masse grasse (figure 1). Après un rappel physiopathologique succinct, nous décrions les interrelations entre exercice physique, fitness et fatness, plus particulièrement l'impact respectif de ces trois composantes sur le risque cardio-métabolique chez la personne adulte non diabétique. Les observations concernant les sujets jeunes, en particulier les adolescents, et les sujets âgés (> 60 ans) seront analysées dans un autre article en raison des spécificités particulières liées à ces deux tranches d'âge [8]. II en sera de même pour l'évaluation des effets de la pratique régulière d'un exercice musculaire, aboutissant à une meilleure aptitude physique, chez la personne ayant une diminution de la tolérance au glucose, et donc à risque de devenir diabétique, et chez le patient présentant déjà un diabète de type 2 (DT2) [9].

\section{Physiopathologie}

Les bénéfices escomptés de la pratique régulière d'une activité physique pour la prévention et le traitement des pathologies cardio-métaboliques associées à l'obésité peuvent s'expliquer par plusieurs mécanismes, dont les effets de l'exercice sur les muscles, en particulier la sensibilité à l'insuline, la graisse, notamment celle à distribution viscérale, l'inflammation larvée et ou encore la réactivité vasculaire (figure 1) [10]. L'ensemble de ces effets aboutit à la diminution bien connue des différents marqueurs/facteurs de risque $\mathrm{CV}$, dont l'hyperglycémie, I'hyperinsulinémie, la dyslipidémie, l'élévation du taux de la C réactive protéine ultrasensible (hsCRP), l'augmentation de pression artérielle,..., bref l'ensemble des variables liées, de près ou de loin, à ce qu'il est convenu d'appeler le syndrome métabolique (SM).

Effets sur le muscle squelettique et la sensibilité à l'insuline

L'exercice physique, indépendamment de toute perte de poids, améliore la sensibilité à l'insuline [10, 11]. En effet, il permet une vasodilatation au sein du muscle strié et augmente l'expression des transporteurs du glucose (GLUT 4) au niveau des membranes cellulaires musculaires, améliorant ainsi la captation périphérique du glucose [12] Une activité physique régulière permet d'augmenter la capacité oxydative des muscles squelettiques, ce qui diminue l'afflux d'acides gras libres vers le foie [12] et réduit l'accumulation de graisse ectopique dans les muscles et les hépatocytes [13]. II en résulte également une amélioration de la sensibilité hépatique à l'insuline [12]. Par ailleurs, l'exercice physique augmente les dépenses caloriques et évite une balance énergétique positive, via l'activation de l'AMP-kinase. En effet, cette enzyme stimule des voies cataboliques impliquées dans la synthèse de l'ATP, telles que la glycolyse et l'oxydation des acides gras, et elle inhibe

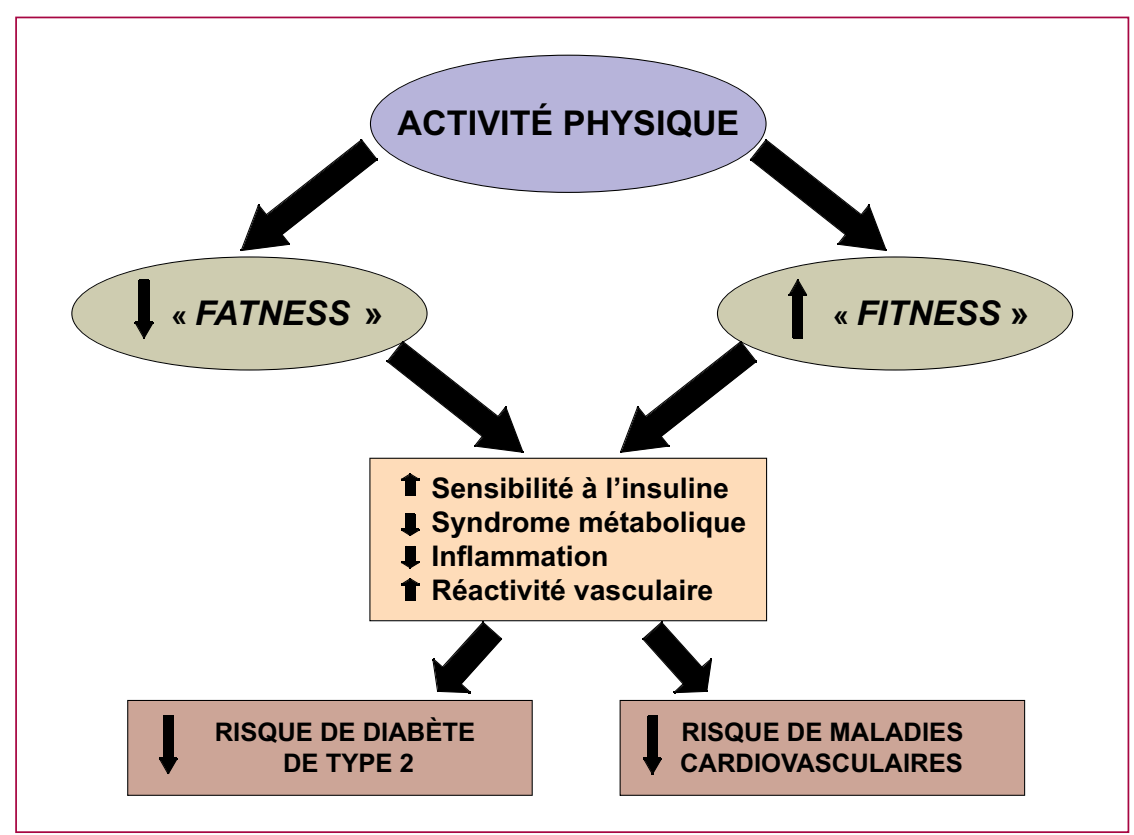

Figure 1 : Illustration des effets conjugués d'un haut degré de fatness et d'un faible degré de fitness sur le risque de développer des maladies métaboliques et cardiovasculaires. La pratique régulière d'une activité physique est susceptible d'influencer favorablement à la fois la fatness et la fitness. 
des voies anaboliques consommatrices d'ATP, à savoir la synthèse des protéines, du cholestérol et des acides gras [14]. Selon Reaven [15], des différences de niveau d'activité physique expliqueraient environ $25 \%$ de la variabilité inter-individuelle de la sensibilité à l'insuline, évaluée essentiellement au niveau musculaire par une technique apparentée au glucose clamp. Une étude canadienne a montré que la bonne forme physique (cardiorespiratory fitness) est associée à une sensibilité à l'insuline élevée, même chez des femmes en surpoids ou obèses ménopausées. Cette relation pourrait être expliquée par des variations de la masse maigre et de puissance musculaire, mais aussi par des modifications de masse grasse viscérale [16].

\section{Effets sur la masse grasse et l'adiposité abdominale}

L'activité physique exerce une influence bénéfique sur le profil métabolique en réduisant les taux de graisse abdominale et de graisse viscérale $[17,18]$. La pratique d'exercice physique d'intensité modérée à élevée, pendant 8 semaines minimum, chez des sujets en surpoids ou obèses est efficace pour réduire la graisse abdominale. De nombreuses études ont confirmé l'existence d'une relation inverse entre le niveau d'activité physique et le tour de taille, le rapport tour de taille/tour de hanche, ainsi que la mesure du taux d'adiposité intraabdominale par différentes techniques d'imagerie médicale (voir plus loin) [17]. Ces effets favorables et les modifications corporelles peuvent survenir même en l'absence de perte pondérale significative [17, 19]. En effet, pour un même indice de masse corporelle (IMC), les individus avec une bonne aptitude physique (fitness) ont moins de graisse abdominale sous-cutanée et de graisse viscérale que les sujets avec un niveau faible de fitness [17, 20]. II a d'ailleurs été montré que la pratique d'une activité physique régulière $(60-85 \%$ de la capacité aérobie maximale, au minimum trois fois par semaine pendant 6 semaines) améliore la sensibilité à l'insuline hépatique, sans nécessaire diminution de la masse grasse totale. II faut, par ailleurs, noter que l'effet favorable sur le profil métabolique est présent indépendam- ment du niveau d'adiposité viscérale ou sous-cutanée [21].

\section{Effets sur les marqueurs inflammatoires}

La pratique régulière d'une activité physique pourrait contribuer à réduire le statut pro-inflammatoire fréquemment rencontré en présence d'une obésité, notamment abdominale, et contribuant à accélérer l'athérosclérose [22]. En effet, un haut niveau d'entraînement cardiorespiratoire diminue les marqueurs de l'inflammation, comme la concentration de hsCRP, indépendamment de toute perte pondérale [23, 24]. Cet effet pourrait contribuer à réduire le taux de morbi-mortalité CV, surtout s'il existe un SM associé à l'obésité [2]. Le rôle de la fatness dans la relation entre fitness et inflammation est particulièrement important chez les femmes, ce qui suggère l'implication des hormones sexuelles [23].

\section{Effets sur la réactivité vasculaire}

L'entraînement physique permet d'améliorer la fonction vasodilatatrice endothélium-dépendante chez les sujets jeunes avec un niveau moyen de fitness [25]. L'amélioration de la vasodilatation endothélium-dépendante résulte d'une élévation des taux de monoxyde d'azote (NO) produit par les cellules endothéliales et une diminution de la sensibilité à l'action vasoconstrictrice de la noradrénaline [26]. Ces effets contribuent, sans doute aussi, au bénéfice de l'exercice physique dans la prévention des maladies $\mathrm{CV}$, en particulier via une diminution de la pression artérielle [25]. II semblerait même que la fitness soit le facteur prédictif prédominant de la fonction endothéliale, plus que les facteurs de risque CV conventionnels et la fatness [27]. Par ailleurs, la pratique d'une activité physique permet d'améliorer la perte de la fonction endothéliale liée à l'âge, en préservant la plasticité vasculaire [28]. Chez les femmes, après la ménopause, un bon niveau de fitness et la pratique régulière d'une activité physique permettent de lutter contre le stress oxydatif et l'hypertension artérielle (HTA), en maintenant en suffisance les enzymes anti-oxydantes et la production de NO [29]. En revanche, dans une autre étude, le niveau de fitness ne semblerait pas modifier l'association entre la fatness et les troubles de réponse vasculaire au stress mental susceptibles d'être impliqués dans la survenue des maladies $\mathrm{CV}$ en relation avec le statut psychosocial [30].

\section{Fatness, activité physique et fitness}

\section{Définition de la fatness}

La fatness se définit par l'ensemble de la masse grasse corporelle. Celle-ci est appréciée le plus simplement par l'IMC, dont la relation avec le risque cardiométabolique a été bien démontrée dans de nombreuses études [31], dont encore récemment dans une analyse combinée de la Physician's Health study et de la Women's Health study [32]. On sait toutefois que de nombreux sujets obèses sont indemnes d'anomalies métaboliques et relativement protégés contre les maladies CV [1], ce qui doit amener à affiner l'approche, par exemple en recourant à la mesure du tour de taille ou du rapport taille/hanches [33]. Cette mesure cible, en effet, mieux l'obésité abdominale et l'adiposité viscérale, les plus délétères sur le plan métabolique, alors que l'adiposité périphérique pourrait, au contraire, plutôt exercer un effet protecteur sur le plan cardio-métabolique, comme nous l'avons discuté précédemment [1]. La mesure du pourcentage de masse grasse, calculé par méthode anthropométrique (mesure des plis cutanés incorporés dans une équation validée), par bioimpédance ou par la méthode Dexa (DXA), ou encore la quantification de la masse grasse localisée en intra-abdominal (tissu adipeux viscéral, stéatose hépatique) par des techniques d'imagerie médicale (CT-scan abdominal, ou résonance magnétique nucléaire de l'abdomen) peuvent représenter d'autres alternatives pour apprécier le degré de fatness d'un individu [34].

Distinction entre activité physique et aptitude cardio-respiratoire

L'activité physique est définie comme tout mouvement du corps produit par les muscles striés squelettiques et qui entraîne une dépense énergétique. La dépense énergétique peut être quantifiée 
en faisant référence au métabolisme basal pris comme unité de base. Un équivalent métabolique (MET) est défini par la quantité d'oxygène consommée lorsqu'un individu est au repos en position assise et équivaut à $3,5 \mathrm{ml} \mathrm{O}_{2}$ par kg de poids corporel et par minute [35]. Le coût énergétique d'une activité physique peut être exprimé en METs et déterminée en divisant la consommation d'oxygène pour réaliser cette activité par 3,5. Malgré ses limites, cette approche pragmatique a le mérite de la simplicité. On estime qu'une activité physique susceptible d'exercer des effets cardiométaboliques bénéfiques doit correspondre à au moins 3-4 METs maintenus pendant au moins 30 minutes et répétés au minimum trois à cinq fois par semaine avec, si possible, au moins un volume d'activité physique de 150 minutes par semaine [11]. II n'existe cependant pas de consensus sur le type d'activité physique susceptible d'exercer les meilleurs effets sur la santé, ainsi que l'a analysé récemment un travail de revue de la littérature comparant les effets d'un exercice continu et ceux d'un exercice fractionné. Ce dernier, sans doute plus facile à réaliser au quotidien, en particulier chez le sujet en surpoids ou obèse, semble, en effet, avoir les mêmes effets bénéfiques qu'un exercice continu sur différents marqueurs de risque métabolique ou vasculaire [36]. Néanmoins, il est démontré que le volume d'activité physique lors d'une période d'entraînement est directement corrélé à l'amélioration de la fitness et que le taux d'absence de réponse diminue avec l'importance du volume imposé [37]. L'activité physique réalisée dans la vie de tous les jours peut aussi s'évaluer à l'aide d'un simple questionnaire, tel que le Questionnaire International d'Activité Physique [38]. De façon plus objective, elle peut aussi être quantifiée par l'utilisation soit d'un accéléromètre, soit d'un podomètre. L'utilisation d'un podomètre a démontré sa capacité à augmenter l'activité physique chez des travailleurs sédentaires et, de façon intéressante, l'augmentation objectivée a été aussi importante chez les personnes avec un IMC élevé (conduisant à une diminution de l'IMC initial, du tour de taille et de la fréquence cardiaque de repos) que chez celles avec un IMC normal [39].
L'activité physique est à différencier de la fitness ou cardiorespiratory fitness, qui représente la condition physique ou l'endurance cardio-respiratoire d'un individu, en relation avec la santé. Celle-ci est déterminée par de nombreux composants individuels (musculaire, cardiaque, respiratoire, métabolique,...) et est influencée à la fois par les gènes et divers facteurs environnementaux, dont prioritairement l'entraînement physique. Généralement, le niveau de fitness s'évalue par un test de consommation maximale d'oxygène $\left(\mathrm{VO}_{2}\right.$ max exprimée en $\mathrm{ml} /$ minute/kg). La $\mathrm{VO}_{2}$ max est un indicateur de la performance potentielle de l'individu dans les épreuves d'endurance. Elle est déterminée par la mesure du volume maximal d'oxygène qui peut être consommé durant un exercice physique d'intensité croissante sur un ergomètre de type tapis roulant ou un ergocyclomètre [40].

\section{Effets respectifs}

\section{des trois composantes}

Un haut degré de fatness et un niveau élevé de fitness exercent des effets cardio-métaboliques en miroir (tableau I). Par ailleurs, la pratique régulière d'une activité physique peut influencer, de façon favorable, à la fois la fatness et la fitness.

Tableau I : Effets cardio-métaboliques en miroir de la fatness et de la fitness.

\begin{tabular}{lcc}
\hline \multicolumn{1}{c}{ Paramètres } & Fatness & Fitness \\
\hline $\begin{array}{l}\text { - Adiposité } \\
\text { viscérale }\end{array}$ & $\uparrow$ & $\downarrow$ \\
$\begin{array}{l}\text { - Stéatose } \\
\text { hépatique }\end{array}$ & $\uparrow$ & $\downarrow$ \\
\hline $\begin{array}{l}\text { - Insulinorésistance } \\
\text { musculaire }\end{array}$ & $\uparrow$ & $\downarrow$ \\
$\begin{array}{l}\text { - Inflammation } \\
\text { silencieuse }\end{array}$ & $\uparrow$ & $\downarrow$ \\
$\begin{array}{l}\text { - Troubles de } \\
\text { réactivité vasculaire }\end{array}$ & $\uparrow$ & $\downarrow$ \\
$\begin{array}{l}\text { - Syndrome } \\
\text { métabolique }\end{array}$ & $\uparrow$ & $\downarrow$ \\
$\begin{array}{l}\text { - Risque de diabète } \\
\text { de type 2 }\end{array}$ & $\uparrow$ & $\downarrow$ \\
\hline $\begin{array}{l}\text { - Risque } \\
\text { cardiovasculaire }\end{array}$ & & \\
\hline
\end{tabular}

Une étude a analysé spécifiquement les effets de l'exercice musculaire, de l'aptitude physique (fitness) et de la fatness sur la sensibilité à l'insuline de différents tissus [12]. Selon ce travail, l'adiposité (fatness) expliquerait la plus grande partie de la variance de la sensibilité à l'insuline globale (60\% expliquée par la fatness, et 2-3\% par la fitness seule) alors que la dépense énergétique liée à l'activité physique rendrait compte de l'essentiel de la variabilité de la sensibilité hépatique, indépendamment de la masse grasse.

L'activité physique et le niveau de fitness sont associés, de façon indépendante, à un moindre risque CV [41] et d'anomalies métaboliques [42]. Les effets de l'exercice physique sur les pathologies CV sont principalement déterminés par une intensité élevée [42]. Un haut niveau d'activité physique réduit les taux de triglycérides et de cholestérol LDL, indépendamment de la fitness [41]. Dans une autre étude, il a été montré qu'une augmentation de la dépense énergétique liée à la pratique d'une activité physique est associée à une réduction du risque métabolique, indépendamment de changement dans le niveau de fatness et de fitness [19]. Par ailleurs, des hauts niveaux de fitness sont associés à une meilleure réduction de tous les facteurs de risque CV pris individuellement ou analysés de façon globale [41, 42]. Ainsi, le niveau de fitness, davantage que l'activité physique seule, est en relation inverse avec le risque $\mathrm{CV}$ [43] et métabolique [42] et ce, indépendamment de la corpulence (fatness) [12]. L'activité physique et le niveau de fitness ont, néanmoins, un rôle indépendant et complémentaire puisque les sujets avec un faible niveau de fitness, restant néanmoins très actifs, ont un risque $\mathrm{CV}$ réduit de $50 \%$ en comparaison avec des sujets inactifs présentant un faible niveau de fitness [41].

\section{Études chez le sujet adulte non diabétique}

Chez l'adulte, l'obésité et le SM sont associés à un risque accru de mortalité de toutes causes et de mortalité $\mathrm{CV}$. Plusieurs études ont démontré une relation inverse entre le niveau de fitness et le 
risque d'émergence d'un SM et de divers facteurs de risque CV $[44,45]$ ou même le risque de mortalité $\mathrm{CV}$ et de mortalité globale [46] et ce, indépendamment du niveau de fatness.

Effets sur la sensibilité à l'insuline, le syndrome métabolique

et les facteurs de risque cardiovasculaire

L'étude européenne RISC (European Relationship between Insulin Sensitivity and Cardiovascular risk) a étudié la relation entre la sensibilité à l'insuline (évaluée par la méthode du clamp euglycémique hyperinsulinique) et l'activité physique, dans une population de 346 hommes et 355 femmes, âgés de 30 à 60 ans, sans pathologies $\mathrm{CV}$ et non traités pour un diabète, une HTA, une dyslipidémie ou une obésité [47]. L'activité physique totale, évaluée sur plusieurs jours, est significativement associée à la sensibilité à l'insuline, aussi bien chez les femmes que chez les hommes. Même parmi les individus les plus sédentaires, une augmentation modeste de l'activité physique améliore la sensibilité à l'insuline de façon significative.

D'après les données de l'étude US National Health and Nutrition Examination Survey 1999-2002 [44], réalisée sur un échantillon de 608 femmes et 692 hommes, âgés de 18 à 49 ans, et ne présentant aucune maladie grave ou incapacité importante, le risque de SM (selon la définition du National Cholesterol Evaluation Program-Adult Treatment Panel III, NCEP-ATP III) chez les hommes est nettement inférieur chez les individus avec une capacité cardio-respiratoire modérée à élevée, comparativement à ceux avec une capacité faible. Chez les femmes, en revanche, il n'y a aucune relation significative entre le SM et la capacité cardio-respiratoire. Dans une autre étude, le score de SM le plus élevé a été trouvé dans la catégorie des sujets obèses avec un niveau de fitness bas, tandis que le score de SM le plus bas a été trouvé dans la catégorie des sujets de poids normal avec un niveau de fitness élevé. Le risque d'augmentation significative du score de SM est plus faible chez les sujets avec un niveau de fitness élevé et ce, à nouveau, indépendamment du niveau de fatness [45].
Une méta-analyse de 24 essais contrôlés a mis en évidence que des sujets en bonne santé, mais sédentaires, qui suivent un programme régulier de marche rapide améliorent de nombreux facteurs de risque $\mathrm{CV}$, en augmentant le cardiorespiratory fitness, en réduisant le poids, I'IMC et le taux de graisse corporelle et en diminuant la pression artérielle diastolique [43].

\section{Effets sur l'athérosclérose carotidienne}

Une étude coréenne, réalisée chez 9871 hommes, âgés de 40 à 81 ans, a montré l'existence d'une relation inverse significative entre le niveau d'aptitude physique et l'athérosclérose carotidienne, évaluée par la mesure de l'épaisseur de l'intimamédia carotidienne par échographie (IMT) [48]. En effet, la présence d'une athérosclérose carotidienne diminue avec l'amélioration du niveau de fitness, passant de $11,7 \%$ chez les sujets avec un niveau faible à $7,7 \%$ chez les sujets avec un niveau élevé. Ceci est également démontré par un rapport de cotes (odds ratio, OR) abaissé à 0,67 [intervalle de confiance à $95 \%$, IC95 \% : 0,55-0,80] lorsqu'on compare la présence d'athérosclérose chez les hommes caractérisés par un haut niveau de fitness par rapport à ceux qui ont un niveau faible. Ces résultats ne changent guère après ajustement pour les facteurs de risque classiques comme l'hypercholestérolémie, I'HTA et le DT2, puisque l'OR ajusté évolue peu, avec une valeur de 0,71 [IC95 \% : 0,59$0,85]$.

\section{Effets sur la mortalité cardiovasculaire et totale}

La sédentarité et un faible niveau de fitness peuvent ainsi être considérés comme des facteurs de risque de mortalité CV. Des études ont démontré que des niveaux modérés et élevés de fitness sont associés à un taux plus faible de mortalité par rapport à un niveau de fitness bas, à la fois chez les sujets de poids normal et chez les sujets avec un excès pondéral $[49,50]$.

Des données observationnelles prospectives de la Aerobics Center Longitudinal Study, réalisée à la clinique de médecine préventive de Dallas, aux États-Unis, ont permis d'analyser les facteurs prédictifs de décès sur une population de 25714 hommes, d'un âge moyen de 44 ans [51]. Les sujets en surpoids ou obèses sont à plus haut risque que les sujets de poids normal. Le risque relatif (RR) de décès CV chez les sujets obèses est au moins aussi élevé chez les sujets avec un faible niveau de fitness (RR $=5,0$ [IC95 \% : $3,6-7,0]$ ) que chez les fumeurs $(R R=4,4$ [IC95 \% : 2,7-7,1]) si l'on prend les sujets de poids normal et non fumeurs comme référence. De même, le $R R$ de décès de toute cause est comparable chez les sujets obèses avec un faible niveau de fitness (RR = 3,1 [IC95 \% : 2,5-3,8]) et chez ceux avec un diabète $(R R=3,1$ [IC95 \% : 2,3-4,2]). Le faible degré de fitness s'avère être un facteur prédictif indépendant de mortalité dans tous les sous-groupes d'IMC, après ajustement pour les autres facteurs de risque. Dans cette population, environ la moitié des hommes obèses ont un faible niveau de fitness, ce qui donne un risque attribuable à la population de $39 \%$ pour la mortalité CV et de $44 \%$ pour la mortalité totale. Cette analyse démontre donc qu'un faible niveau de fitness est un facteur de risque indépendant de mortalité CV et totale aussi puissant que d'autres facteurs de risque reconnus jouer un rôle majeur, comme le tabagisme ou le diabète [51].

Dans la vaste étude canadienne de Katzmarzyk et al. [46], réalisée sur une population de 19173 hommes, âgés de 20 à 83 ans (âge moyen : 43 ans), les risques de mortalité totale et de mortalité CV associés au poids corporel et au $\mathrm{SM}$ ne sont plus significatifs lorsqu'on ajuste pour la capacité cardio-respiratoire. En effet, le RR de mortalité globale est significativement plus élevé chez les sujets obèses avec $\mathrm{SM}(\mathrm{RR}=1,55)$ que chez les sujets de poids normal sans SM, et cette différence disparaît lorsqu'on inclut la capacité cardio-respiratoire comme covariable $(R R=0,93)$. De même, le risque de mortalité CV est plus élevé chez les sujets en surpoids avec $S M(R R=1,80)$ et les sujets obèses avec $S M(R R=2,83)$ et même sans SM $(R R=2,70)$, en comparaison aux sujets de poids normal sans SM. Cependant, après introduction de la capacité cardiorespiratoire comme covariable, tous les RR estimés pour la mortalité CV sont 
atténués et ne sont plus significativement différents de la valeur de référence « 1 » attribuée au groupe de sujets de poids normal sans SM. Par ailleurs, une capacité cardio-respiratoire élevée chez des sujets avec un SM est associée significativement à un moindre risque de mortalité globale $(R R=0,81)$ et de mortalité CV $(R R=0,74)$. Ainsi, la capacité cardiorespiratoire atténue les effets du SM sur le risque de mortalité $\mathrm{CV}$ et globale dans toutes les catégories d'IMC [46].

Une étude finlandaise, incluant un suivi de 16 ans chez 6787 sujets, âgés de 19 à 63 ans, a montré qu'un niveau faible d'exercice physique et de fitness est associé à une augmentation du risque de mortalité et ce, après ajustement pour d'autres facteurs de risque [52]. Ainsi, parmi les 1122 femmes étudiées, celles qui sont les moins actives ont un RR de mortalité CV de 1,61 [IC95 \% : $0,98-2,64]$ par rapport à celles qui sont actives ; chez les 1090 hommes étudiés, le RR de mortalité coronarienne s'élève à 1,66 [IC95 \% : 0,92-2,98] chez les sédentaires comparés aux actifs. Dans cette étude, le niveau d'exercice physique est plus discriminant en tant que facteur pronostic de mortalité que I'IMC. Les effets bénéfiques de la pratique régulière d'un exercice physique sont semblables chez les sujets obèses et non obèses et sont également objectivés indépendamment du niveau de fitness initial.

Une étude américaine récente, réalisée chez 13155 hommes avec HTA, a montré que le niveau de fitness, évalué lors d'un test d'effort standardisé, est un puissant modulateur de l'association entre l'adiposité et le risque de mortalité dans cette population particulière. Ainsi, un haut niveau de fitness est capable d'annuler la relation habituelle entre la mortalité, de toutes causes ou d'origine $C V$, et l'obésité, que celle-ci soit évaluée par l'IMC, le tour de taille ou le pourcentage de masse grasse [53]. II en est de même dans la population coronarienne. Ainsi, les influences respectives de l'aptitude physique (mesurée lors d'un test d'effort) et de l'adiposité (estimée simplement par l'IMC) sur la mortalité globale ont été analysées récemment dans une population de 12417 hommes, âgés de 40 à 70 ans, présentant une suspicion ou une confirmation de maladie coronarienne [54]. Après un suivi de 7,7 années, les sujets en surpoids avec le plus haut niveau d'aptitude physique se sont avérés avoir la mortalité la plus basse $(R R=0,4)$ et les sujets en déficit pondéral relatif avec le niveau le plus bas

\section{Les points essentiels}

- Les effets délétères d'un excès de masse grasse (fatness) pourraient être contrecarrés par la pratique régulière d'exercices aboutissant à une bonne forme physique (fitness).

- La pratique régulière d'une activité physique améliore la sensibilité (musculaire hépatique) à l'insuline, contribue à réduire la masse grasse (abdominale), diminue les marqueurs inflammatoires et améliore la réactivité vasculaire.

- Un haut degré de fatness et un niveau élevé de fitness exercent des effets cardio-métaboliques en miroir. La pratique régulière d'une activité physique a un effet bénéfique à la fois sur la fatness et la fitness. Les trois composantes peuvent influencer de façon indépendante le risque cardiovasculaire.

- Chez le sujet adulte non diabétique, de grandes enquêtes ont montré un effet positif du niveau d'aptitude physique sur le niveau de sensibilité à l'insuline et les marqueurs du syndrome métabolique, y compris dans une population en surpoids ou obèse.

- Chez le sujet adulte non diabétique : un bon niveau d'aptitude physique atténue remarquablement le risque de morbi-mortalité cardiovasculaire et ce, quel que soit le degré d'indice de masse corporelle.

- Ces observations plaident pour une prise en charge globale, visant à la fois à prévenir/ traiter l'obésité et à améliorer l'aptitude physique par la pratique régulière d'exercices musculaires.

d'aptitude physique, la mortalité la plus forte $(R R=4,5)$, par rapport au groupe contrôle des hommes de poids normal avec une haute aptitude physique. Les sujets en surpoids ou obèses avec un relativement bon niveau d'aptitude physique ont eu une survie non différente des sujets témoins. Ainsi, le niveau d'aptitude physique explique le paradoxe apparent de la relation obésité-mortalité puisque les sujets en surcharge pondérale ou obèse n'ont une espérance de vie accrue que s'ils ont un haut niveau de fitness.

Un faible niveau d'aptitude physique, quantifiée par une valeur basse de $\mathrm{VO}_{2} \mathrm{max}$ et/ou une récupération postexercice lente de la fréquence cardiaque, est un facteur prédictif majeur et indépendant de morbidité et de mortalité CV. Ceci est bien illustré et remarquablement documenté dans une revue systématique de 36 publications parues depuis 1990 [4]. La majorité des études analysées confirme que le risque de mortalité globale et CV est plus faible chez les sujets en excès pondéral et avec obésité modérée (IMC $\leq 35 \mathrm{~kg} / \mathrm{m}^{2}$ ) avec un bon niveau de fitness, en comparaison avec des sujets avec un IMC normal et un niveau de fitness bas. Les différences apparaissent moins nettement, et de façon non significative, si l'on prend en compte non pas le niveau de fitness, mais plutôt le simple niveau d'activité physique, selon les définitions mentionnées précédemment.

\section{Effets d'une prise en charge globale}

Si un haut niveau de fatness et un bas niveau de fitness représentent autant de facteurs de risque CV, il est important de proposer une approche globale ciblant les deux facteurs de risque simultanément, dans toute la mesure du possible $[55,56]$. La prise en charge de l'obésité dans une optique de réduction du risque a fait l'objet de recommandations collégiales par plusieurs sociétés américaines réputées [31]. Par ailleurs, l'American College of Sports Medicine et l'American Heart Association ont aussi émis des recommandations conjointes pour promouvoir l'activité physique dans une optique de santé publique [6]. Un plaidoyer a été publié récemment pour 
que l'Europe développe également des recommandations pour promouvoir l'activité physique dans un souci d'une amélioration de la santé [57].

Chez les personnes adultes, il est évident que le maintien d'un bon niveau d'activité physique reste une approche efficace dans la prévention primaire des maladies métaboliques et CV [19]. Le contrôle et la réduction de l'excès de masse grasse ne doivent néanmoins pas être négligés puisque les sujets avec un IMC élevé et un haut niveau d'activité physique ont plus de risque de développer un DT2 et de présenter des facteurs de risque $\mathrm{CV}$ que les sujets avec un IMC normal et un faible niveau d'activité physique [4]. Les patients qui sont « fat », mais « fit », nécessitent donc une prise en charge permettant une perte pondérale afin de diminuer davantage leur risque CV [58]. Une étude récente a montré que le manque d'aptitude physique (low fitness) et l'adiposité abdominale évaluée par le tour de taille (high fatness) agissent tous les deux comme facteurs de risque $\mathrm{CV}$ et ce, de façon indépendante [59]. En pratique clinique, il est donc important d'évaluer simultanément le niveau de fitness et le tour de taille comme marqueur de l'obésité abdominale. Néanmoins, cette étude démontre que ni une bonne aptitude physique, ni un statut mince ne permettent de réduire le risque lié au tabagisme, facteur de risque classique majeur bien connu. Une nouvelle publication récente de la Aerobics Center Longitudinal Study, déjà citée, analysant 2642 décès lors d'un suivi de 16 années de 38110 hommes, âgés de 16 à 84 ans, donne des arguments supplémentaires pour une approche globale [60]. Ainsi, il apparaît évident que chaque facteur protecteur joue un rôle positif et que le cumul de cinq facteurs évalués (IMC normal, absence de tabagisme actif, consommation modérée d'alcool, haut niveau d'aptitude cardiorespiratoire et pratique régulière d'une activité physique) procure la meilleure protection avec un taux de mortalité ajusté de seulement 0,39 [IC95\%: $0,31-0,48]$ par rapport aux sujets n'ayant aucun de ces facteurs protecteurs.

\section{Conflits d'intérêt}

Les auteurs n'ont aucun conflit d'intérêt en relation avec le contenu de cet article.

\section{Références}

[1] Esser N, Paquot N, Scheen AJ. Sujets « métaboliquement sains ", bien qu'obèses. $1^{\text {re }}$ partie : diagnostic, physiopathologie et prévalence. Obésité 2009;4:56-65.

[2] Katzmarzyk PT, Church TS, Blair SN. Cardiorespiratory fitness attenuates the effects of the metabolic syndrome on all-cause and car-

\section{Conclusion}

L'exercice physique exerce des effets bénéfiques sur le profil métabolique et le risque cardiovasculaire. II améliore la sensibilité à l'insuline par ses actions sur le muscle squelettique et sur la graisse viscérale, et il réduit le statut pro-inflammatoire lié à l'obésité, tout en améliorant la réactivité vasculaire. La pratique régulière d'une activité physique contribue, par ailleurs, à élever le niveau de fitness, lui-même inversement corrélé avec le risque cardiovasculaire. Ainsi, un bon niveau d'aptitude physique permet de contrecarrer - voire même d'annuler - les conséquences néfastes d'un excès de masse grasse. Chez le sujet adulte non diabétique, un bon niveau de fitness est associé à un moindre développement de troubles métaboliques (syndrome métabolique) et à une réduction du risque de mortalité globale et cardiovasculaire. Trop souvent, on constate que l'excès pondéral est associé à une moins bonne aptitude physique. II est donc important d'encourager les personnes à rester actives, le maintien d'une activité physique régulière ayant une place primordiale dans la prévention primaire des pathologies cardio-métaboliques et ce, indépendamment du niveau de fatness. Nous envisagerons dans deux prochains articles, les effets spécifiques des interrelations fatness-fitness chez les personnes jeunes ou âgées [8], ainsi que cette problématique plus spécifiquement dans la population présentant une diminution de la tolérance au glucose ou un diabète de type 2 [9]. diovascular disease mortality in men. Arch Intern Med 2004;164:1092-7.

[3] Lee DC, Sui X, Blair SN. Does physical activity ameliorate the health hazards of obesity? $\mathrm{Br} J$ Sports Med 2009;43:49-51.

[4] Fogelholm M. Physical activity, fitness and fatness: relations to mortality, morbidity and disease risk factors. A systematic review. Obes Rev 2009 Sep 9: [Epub ahead of print].

[5] Olds TS, Ridley K, Tomkinson GR. Declines in aerobic fitness: are they only due to increasing fatness? Med Sports Sci 2007;50:226-40.

[6] Haskell WL, Lee IM, Pate RR, et al. Physical activity and public health: updated recommendation for adults from the American College of Sports Medicine and the American Heart Association. Med Sci Sports Exerc 2007;39:1423-34.

[7] Esser N, Paquot N, Scheen AJ. Sujets « métaboliquement sains ", bien qu'obèses. 2ème partie : Pronostic et prise en charge. Obésité 2009;4:134-41.

[8] Esser N, Paquot N, Scheen AJ. Aptitude physique versus adiposité : impacts métaboliques respectifs chez l'enfant/adolescent et chez la personne âgée. Médecine des maladies métaboliques 2010 : in press.

[9] Esser N, Paquot N, Scheen AJ. Aptitude physique versus adiposité : impacts cardio-métaboliques respectifs chez le sujet adulte obèse avec diminution de tolérance au glucose ou diabète de type 2. Médecine des maladies métaboliques 2010 : in press

[10] Wang Y, Simar D, Fiatarone Singh MA. Adaptations to exercise training within skeletal muscle in adults with type 2 diabetes or impaired glucose tolerance: a systematic review. Diabetes Metab Res Rev 2009;25:13-40.

[11] Scheen AJ, Paquot N. Effets bénéfiques de l'activité physique sur les facteurs de risque cardio-vasculaire. Rev Med Liège 2001;56:239-43.

[12] Holt HB, Wild $\mathrm{SH}$, Wareham $\mathrm{N}$, et al. Differential effects of fatness, fitness and physical activity energy expenditure on whole-body, liver and fat insulin sensitivity. Diabetologia 2007;50:1698-706.

[13] Freedland ES. Role of a critical adipose tissue threshold (CVATT) in metabolic syndrome: implication of controlling dietary carbohydrates: a review. Nutr Metab (Lond) 2004;1:12.

[14] Viollet B, Guigas B, Leclerc H, et al. AMPactivated protein kinase in the regulation of hepatic energy metabolism: from physiology to therapeutic perspectives. Acta Physiol (Oxf) 2009;19:681-98.

[15] Reaven G. All obese individuals are not created equal: insulin resistance is the major determinant of cardiovascular disease in overweight/obese individuals. Diab Vasc Dis Res 2005;2:105-12.

[16] Messier V, Malita FM, Rabasa-Lhoret R, et al. Association of cardiorespiratory fitness with insulin sensitivity in overweight and obese postmenopausal women: a Montreal Ottawa New Emerging Team study. Metabolism 2008;57:1293-8.

[17] Kay SJ, Fiatarone Singh MA. The influence of physical activity on abdominal fat: a systematic review of the literature. Obes Rev 2006;7:183-200. 
[18] Ohkawara K, Tanaka S, Miyachi M, et al. A dose-response relation between aerobic exercise and visceral fat reduction: systematic review of clinical trials. Int J Obes (Lond) 2007;31:1786-97 [Erratum in: Int J Obes (Lond) 2008;32:395].

[19] Ekelund U, Franks PW, Sharp S, et al. Increase in physical activity energy expenditure is associated with reduced metabolic risk independent of change in fatness and fitness. Diabetes Care 2007;30:2101-6.

[20] O'Donovan G, Thomas EL, McCarthy JP, et al. Fat distribution in men of different waist girth, fitness level and exercise habit. Int J Obes (Lond) 2009;33:1356-62.

[21] Lee S, Kuk JL, Katzmarzyk PT, et al. Cardiorespiratory fitness attenuates metabolic risk independent of abdominal subcutaneous and visceral fat in men. Diabetes Care 2005;28:895-901.

[22] Rocha VZ, Libby P. Obesity, inflammation, and atherosclerosis. Nat Rev Cardiol 2009;6:399-409.

[23] Hamer M. The relative influences of fitness and fatness on inflammatory factors. Prev Med 2007;44:3-11.

[24] Campbell PT, Campbell KL, Wener MH, et al. A yearlong exercise intervention decreases CRP among obese postmenopausal women. Med Sci Sports Exerc 2009;41:1533-9.

[25] Clarkson P, Montgomery HE, Mullen MJ, et al. Exercise training enhances endothelial function in young men. J Am Coll Cardiol 1999;33:1379-85.

[26] Delp MD. Effects of exercise training on endothelium-dependent peripheral vascular responsiveness. Med Sci Sports Exerc 1995;27:1152-7.

[27] Lippincott MF, Carlow A, Desai A, et al. Relation of endothelial function to cardiovascular risk in women with sedentary occupations and without known cardiovascular disease. Am J Cardiol 2008;102:348-52.

[28] Wray DW, Uberoi A, Lawrenson L, Richardson RS. Evidence of preserved endothelial function and vascular plasticity with age. Am J Physiol Heart Circ Physiol 2006;290:H1271-7.

[29] Pialoux V, Brown AD, Leigh R, et al. Effect of cardiorespiratory fitness on vascular regulation and oxidative stress in postmenopausal women. Hypertension 2009;54:1014-20.

[30] Hamer M, Boutcher YN, Boutcher SH. Fatness is related to blunted vascular stress responsivity, independent of cardiorespiratory fitness in normal and overweight men. Int J Psychophysiol 2007;63:251-7.

[31] Klein S, Burke LE, Bray GA, et al; American Heart Association Council on Nutrition, Physical Activity, and Metabolism. Clinical implications of obesity with specific focus on cardiovascular disease: a statement for professionals from the American Heart Association Council on Nutrition, Physical Activity, and Metabolism: endorsed by the American College of Cardiology Foundation. Circulation 2004;110:2952-67.

[32] Gelber RP, Gaziano JM, Orav EJ, et al. Measures of obesity and cardiovascular risk among men and women. J Am Coll Cardiol 2008;52:605-15.

[33] Klein S, Allison DB, Heymsfield SB, et al; Association for Weight Management and Obesity Prevention; NAASO; Obesity Society; American Society for Nutrition; American Diabetes Association. Waist circumference and cardiometabolic risk: a consensus statement from shaping America's health: Association for Weight Management and Obesity Prevention; NAASO, the Obesity Society; the American Society for Nutrition; and the American Diabetes Association. Diabetes Care 2007;30:1647-52.

[34] Fogelholm M, van Marken Lichtenbelt W. Comparison of body composition methods: a literature analysis. Eur J Clin Nutr 1997;51:495-503.

[35] Sidney JM, Blümchen G. Metabolic equivalents (METS) in exercise testing, exercise prescription, and evaluation of functional capacity. Clin Cardiol 1990;13:555-65.

[36] Murphy MH, Blair SN, Murtagh EM. Accumulated versus continuous exercise for health benefit: a review of empirical studies. Sports Med 2009;39:29-43.

[37] Sisson SB, Katzmarzyk PT, Earnest CP, et al. Volume of exercise and fitness nonresponse in sedentary, postmenopausal women. Med Sci Sports Exerc 2009;41:539-45.

[38] Papathanasiou G, Georgoudis G, Georgakopoulos D, et al. Criterion-related validity of the short International Physical Activity Questionnaire against exercise capacity in young adults. Eur J Cardiovasc Prev Rehabil 2009 Nov 24: [Epub ahead of print].

[39] Chan CB, Ryan DA, Tudor-Locke C. Health benefits of a pedometer-based physical activity intervention in sedentary workers. Prev Med 2004;39:1215-22.

[40] Jouannot P. VO2 max: technique et importance pratique. Presse Méd 2001;30:835-40.

[41] Ekblom-Bak E, Hellénius ML, Ekblom O, et al. Independent associations of physical activity and cardiovascular fitness with cardiovascular risk in adults. Eur $\mathrm{J}$ Cardiovasc Prev Rehabil 2010;17:175-80.

[42] Sassen B, Cornelissen VA, Kiers H, et al. Physical fitness matters more than physical activity in controlling cardiovascular disease risk factors. Eur J Cardiovasc Prev Rehabil 2009;16:677-83.

[43] Murphy MH, Nevill AM, Murtagh EM, Holder $\mathrm{RL}$. The effect of walking on fitness, fatness and resting blood pressure: a meta-analysis of randomised, controlled trials. Prev Med 2007:44:377-85.

[44] Brien SE, Janssen I, Katzmarzyk PT. Cardiorespiratory fitness and metabolic syndrome: US National Health and Nutrition Examination Survey 1999-2002. Appl Physiol Nutr Metab 2002;32:143-7

[45] Church TS, Finley CE, Earnest CP, et al. Relative associations of fitness and fatness to fibrinogen, white blood cell count, uric acid and metabolic syndrome. Int $\mathrm{J}$ Obes Relat Metab Disord 2002;26:805-13.

[46] Katzmarzyk PT, Church TS, Janssen I, et al. Metabolic syndrome, obesity, and mortality: impact of cardiorespiratory fitness. Diabetes Care 2005;28:391-7.

[47] Balkau B, Mhamdi L, Oppert JM, et al; EGIRRISC Study Group. Physical activity and insulin sensitivity. The RISC Study. Diabetes 2008;57:2613-8.

[48] Lee CD, Jae SY, Iribarren C, et al. Physical fitness and carotid atherosclerosis in men. Int $J$ Sports Med 2009;30:672-6.

[49] Lee CD, Jackson AS, Blair SN. US weight guidelines: is it also important to consider cardiorespiratory fitness? Int J Obes Relat Metab Disord 1998;22(Suppl.2):S2-S7.

[50] Barlow CE, Kohl HW $3^{\text {rd }}$, Gibbons LW, Blair SN. Physical fitness, mortality and obesity. Int J Obes Relat Metab Disord 1995;19(Suppl.4):S41-S44.

[51] Wei M, Kampert JB, Barlow CE, et al. Relationship between low cardiorespiratory fitness and mortality in normal-weight, overweight, and obese men. JAMA 1999;282:1547-53.

[52] Haapanen-Niemi N, Milunpalo S, Pasanen $M$, et al. Body mass index, physical inactivity and low level of physical fitness as determinants of all-cause and cardiovascular disease mortality-16 y follow-up of middle-aged and elderly men and women. Int J Obes Relat Metab Disord 2000;24:1465-74.

[53] McAuley PA, Sui X, Church TS, et al. The joint effects of cardiorespiratory fitness and adiposity on mortality risk in men with hypertension. Am J Hypertens 2009;22:1062-9.

[54] McAuley PA, Kokkinos PF, Oliveira RB, et al. Obesity paradox and cardiorespiratory fitness in 12,417 male veterans aged 40 to 70 years. Mayo Clin Proc 2010;85:115-21.

[55] Jakicic JM. The effect of physical activity on body weight. Obesity (Silver Spring) 2009;17(Suppl.3):S34-8.

[56] Larson-Meyer DE, Redman L, Heilbronn LK, et al. Caloric restriction with or without exercise: the fitness versus fatness debate. Med Sci Sports Exerc 2010;42:152-9.

[57] Oja P, Bull FC, Fogelholm M, Martin BW. Physical activity recommendations for health: what should Europe do? BMC Public Health 2010;10:10.

[58] Diaz VA, Player MS, Mainous AG 3rd, et al. Competing impact of excess weight versus cardiorespiratory fitness on cardiovascular risk. Am J Cardiol 2006;98:1468-71.

[59] Ekblom-Bak E, Hellenius ML, Ekblom O, et al. Fitness and abdominal obesity are independently associated with cardiovascular risk. J Intern Med 2009;266:547-57.

[60] Byun W, Sieverdes JC, Sui X, et al. Effect of positive health factors and all-cause mortality in men. Med Sci Sports Exerc 2010; Feb 4. [Epub ahead of print]. 